\title{
ANTIMICROBIAL STEWARDSHIP IN VETERINARY MEDICINE: IN NEED OF URGENT IMPLEMENTATION
}

\author{
I. SAMANTA*, S. BANDYOPADHYAY ${ }^{1}$, T. K. DUTTA² AND S. N. JOARDAR \\ Department of Veterinary Microbiology \\ West Bengal University of Animal and Fishery Sciences \\ Belgachia, Kolkata- 700 037, West Bengal, India
}

\begin{abstract}
Antimicrobial resistance (AMR) has emerged as a major public health problem and is considered as one of the greatest threats to human and animal health. To prevent emergence and spread of health-care associated antimicrobial resistant organisms, formulation of strategies are quite essential of which antimicrobial stewardship remains most vital. 'Antimicrobial stewardship' can be defined as a 'coherent set of actions' which confirms effective therapeutic availability of antimicrobials during necessity. Formation of a national veterinary stewardship policy is required with species wise therapeutic guidelines, formulation of online and offline courses for veterinarians and para-veterinarians, monitoring of antibiotic use and surveillance of current antibiotic resistance pattern in animal/fish associated bacteria. Good stewardship practice (GSP) is collection of active and motivated exercises for judicious antimicrobial use. The GSP consists of ' $5 R$ ' i.e. responsibility, reduction, refinement, replacement and review. Indian Council of Agriculture Research in collaboration with FAO framed 'Indian network for fishery and animal antimicrobial resistance (INFAAR)', a national network of veterinary laboratories to generate data on antimicrobial resistance specific to livestock and fisheries.
\end{abstract}

Key words: Antimicrobial resistance, INFAAR, Stewardship, Veterinary medicine

By the mid-20 th century discovery of antibacterial drugs resulted in to dramatic improvement in human and animal health, and productivity so much, so that scientists and planners entered in euphoria that bacterial diseases would be considered as historic events within a short time. However, that euphoria didn't exist long as bacteria started adopting clever mechanisms and showing its manifestation of resistance against various newly developed drugs also. The resistance mechanisms pose a great threat to the health issues in both human and animals. Antimicrobial resistance (AMR) has now emerged as a major public health problem throughout the globe. The resistant

*Corresponding Author

${ }^{1}$ ICAR-Indian Veterinary Research Institute, Eastern Regional Station, Kolkata

${ }^{2}$ Department of Veterinary Microbiology, Central Agricultural University, Aizawl, Mizoram 
bacteria causing different infections cannot be eliminated easily as limited therapeutic options exists that lead to prolonged illness, even death. Longer period of infectivity associated with treatment failure increases the chance of exposure of resistant microbes to the population. Emergence of multi-drug resistant microorganisms due to misuse and abuse of drugs created the situation more complex.

\section{Possible way out to combat antimicrobial resistance}

In 2006, Centre for disease control and prevention, CDC (USA) guideline stated that emergence of multi-drug resistance can be controlled in paying great attention to judicious antimicrobial use. Subsequently, in 2009 CDC launched 'Get smart for healthcare campaign' to promote improved use of antibiotic in in- patient settings. In 2015, the White House published the national plan for combating antibioticresistant bacteria with five main goals to $-i$ ) Curb antibiotic resistance, ii) Strengthen antimicrobial resistance surveillance, iii) Advance development of rapid diagnostics, iv) Accelerate research targeting novel therapeutics and v) collaborate with other countries to strengthen prevention, development and surveillance efforts (Antimicrobial Stewardship Programme Guideline, Indian Council of Medical Research, 2018).

As per World Health Organization (WHO) antimicrobial resistance is one of the greatest threats to human health, whereas about $50 \%$ of antimicrobial use has been found to be inappropriate. Hence, to prevent emergence and spread of health-care associated antimicrobial resistant organisms, formulation of strategies are essential. Successive implementation of the effective strategies encompasses a) effective clinical antimicrobial stewardship, b) meticulous hand hygiene and c) surveillance and monitoring of antimicrobial resistance, especially multidrug resistant (MDR) organisms. Antimicrobial stewardship includes the evidence based education of physicians about appropriate drug, dose, dosing interval and duration. De-escalation and optimizing campaigns should be taken to educate and inform the public about the specific indications for antibiotic use, origin and spread of antimicrobial resistance and what needs to be done to control it (Antimicrobial Stewardship Programme Guideline, Indian Council of Medical Research, 2018).

\section{Definition and utility of antimicrobial stewardship}

'Antimicrobial stewardship' can be defined as a 'coherent set of actions' which confirms effective therapeutic availability of antimicrobials during necessity (Dyar et al., 2017). The 'coherent set of actions' incorporates the therapeutical knowhow i.e. selection, dose, route of administration and duration of antibiotics to a concept of responsibility (Goff, 2011). This coordinated intervention is designed to improve and measure the appropriate use of antimicrobial agents by promoting the selection of optimal antimicrobial drug regimen including dosing, duration of therapy and route of administration as per the Infectious Diseases of America (IDSA), the Society for Healthcare Epidemiology of America (SHEA) and the Pediatric Infectious Diseases Society (PIDS) (Fishman, 2012). 
The aim of the stewardship programme includes an approach to judicious antibiotic use in patients minimizing the toxicity and suitable conditions for the generation of resistant bacteria. It also encompasses a Government initiated multifaceted approach including policies, guidelines, surveillance of resistant organisms, mass awareness campaign and audit of practice.

The goal of antimicrobial stewardship is 3fold. The first goal is to work with health care practitioners to help each patient receive the most appropriate antimicrobial with the correct dose and duration. The Optimal antimicrobial therapy rests upon 4D's, viz. right drug, right dose, de-escalation to pathogen-directed therapy and right duration of therapy (Joseph and Rodvold, 2008). The second goal is to prevent antimicrobial overuse, misuse and abuse. The third goal is to minimize the development of resistance (Doron and Davidson, 2011).

Implementation of stewardship program in veterinary medicine can decrease the occurrence of resistance, antibiotic associated adverse effects and treatment costs besides improving clinical success as observed in human studies (Deuster et al., 2010; Toth et al., 2010; Davey et al., 2013).

\section{Strategy}

Formation of a national veterinary stewardship policy is required with species wise therapeutic guidelines, formulation of online and offline courses for veterinarians (may be under continuing veterinary education program) and paraveterinarians, monitoring of antibiotic use and surveillance of current antibiotic resistance pattern in animal/fish associated bacteria (Hardefeldt et al., 2018). The stewardship intervention in animal/fishery sector is difficult to implement due to insufficient numbers of veterinarians or fishery officers, limited access to education and training, limited communication between academic and community veterinarians/fishery officers, restricted diagnostic tools and laboratory support, competition between veterinary practitioners, and sales of antibiotics without consultation and prescription. Even in developed countries such as in United States little laboratory support is available for stewardship program and data of antibiotic consumption for companion and food animals is not available (Grayzel et al., 2015). In US antimicrobial stewardship is considered as a threat to meat industry in the forms of affordability of meat, consistency of supply chain, and profits (D'Angeli et al., 2016).

\section{Good stewardship practice (GSP)}

Good stewardship practice (GSP) is collection of active and motivated exercises for judicious antimicrobial use (Prescott and Boerlin, 2016). The GSP consists of ' $5 R$ ' i.e. responsibility, reduction, refinement, replacement and review. In veterinary practices, 'responsibility' is a mutual approach between the practitioner with the selection of a proper antibiotic and the owner by following the instructions for proper use of the antibiotic and other management practices (Page et al., 2014). 'Reduction' is defined as improvement of biosecurity strategy, use of vaccine, providing balanced feed, maintenance of proper hygiene, selection of genetically disease resistant flock which can minimize the infectious diseases and subsequent use of antibiotics (Swaggerty 
et al., 2014; Murphy et al., 2017). Introduction of biosecurity practices among the backyard poultry farmers in West Bengal reduced the infection rate in birds and improved the egg production (Samanta et al., 2015, 2018). 'Refinement' is the accurate diagnosis of an infection of bacterial origin followed by prescription of the appropriate drug in proper dose, and route, and for the exact period. 'Replacement' is the use of alternative approach in place of antibiotics if sufficient evidence is there for efficacy and safety. The alternative approach in livestock and poultry includes use of probiotics, prebiotics, synbiotics, bacteriophages, essential oils, antimicrobial peptides, immunoglobulins, spray dried immune plasma, teat sealing paste during dry period and artificial bovine cytokines (Torrallardona, 2009; Ghosh et al., 2012; Gadde et al., 2015; Chowdhury et al., 2018). 'Review' is a continuous process of evaluation for all the interventions taken. Progress is measured for each objective with the available data associated with antibiotic consumption in food or companion animals, correlation between the consumption and resistance pattern in the bacteria, quality of antibiotics used, control of infectious diseases, and moreover, improvement of each intervention (Dunn and Dunn, 2012; Kallen and Prins, 2017). The example of GSP was detected in Netherlands which reported 58\% decrease in antibiotic sales in animal sector during 200915 and it was associated with reduced

\section{REFERENCES}

Antimicrobial Stewardship Programme Guideline, Indian Council of Medical Research, 2018. Available at: https://icmr.nic.in/sites/ default/files/guidelines/AMSP_0.pdf, accessed on $5 / 11 / 19$ antimicrobial resistance in broilers, veal calves, and pigs (ECDGHFS, 2017).

\section{Guidelines to minimize antimicrobial resistance}

In India, Prevention of Food Adulteration Rules, 1995-part XVIII prescribed limit for antibiotics to be used in fisheries. Food safety and standards authority of India (FSSAI) has framed the regulations to ban the use of certain antibiotics and pharmacologically active substances in fisheries (NAP-AMR, 2017-21). Indian Council of Agriculture Research (ICAR) in collaboration with FAO framed a national network of veterinary laboratories to generate data on antimicrobial resistance specific to livestock and fisheries. The network is known as 'Indian network for fishery and animal antimicrobial resistance (INFAAR)'. Currently, the network has 21 members from ICAR institutions and agricultural/veterinary Universities. Based on the data generated out of INFAAR activities it is hoped that successful strategies like antimicrobial stewardship in veterinary medicine will be given a shape for Indian perspective to address and mitigate the AMR issues in future.

\section{ACKNOWLEDGEMENT}

The authors are thankful to the Vice Chancellor, West Bengal University of Animal and Fishery Sciences, Kolkata for providing necessary infrastructure facilities.

Chowdhury S, Mandal GP, Patra AK, Kumar P, Samanta I et al., 2018. Different essential oils in diets of broiler chickens: 2 . Gut microbes and morphology, immune response, and some blood profile and 
antioxidant enzymes. Anim Feed Sci Tech, 236: $39-47$

D'Angeli MA, Baker JB, Call DR, Davis MA, Kauber KJ et al., 2016. Antimicrobial stewardship through a one health lens: observations from Washington State. Int J Hlth Govern, 21: 114-130

Davey P, Brown E, Charani E, Fenelon L, Gould IM et al., 2013. Interventions to improve antibiotic prescribing practices for hospital inpatients. Cochrane Database Syst Rev, 30: CD003543

Deuster S, Roten I and Muehlebach S, 2010. Implementation of treatment guidelines to support judicious use of antibiotic therapy. J Clin Pharm Ther, 35: 71-78

Doron S and Davidson LE, 2011. Antimicrobial stewardship. Mayo Clin Proc, 86: 1113-1123

Dunn F and Dunn J, 2012. Clinical audit: Application in small animal practice. In Practice, 34: 243-245

Dyar OJ, Huttner B, Schouten J and Pulcini C, 2017. What is antimicrobial stewardship? Clin Microbiol Infect, 23: 793-798

European Commission Directorate-General for Health and Food Safety (ECDGHFS), 2017. Final report of a fact-finding mission carried out in the Netherlands from 13 September 2016 to 20 September 2016 in order to gather information on the prudent use of antimicrobials in animals. Audit number 2016-8889, http://ec.europa.eu/food/ a udits-analysis / a udit_reports / details.cfm?rep_id= 3753. Accessed on September 11, 2019

Fishman N, 2012. Policy statement on amtimicrobial stewardship by the Society for Healthcare Epidemiology of America (SHEA), the Infectious Diseases Society of America (IDSA), and the Pediatric Diseases Society (PIDS). Infect Control Hosp Epidemiol, 33: 322-327

Gadde U, Rathinam T and Lillehoj HS, 2015. Passive immunization with hyperimmune egg-yolk IgY as prophylaxis and therapy for poultry diseases-A review. Anim Hlth Res Rev, 16: 163-176

Ghosh TK, Haldar S, Bedford MR, Muthusami N and Samanta I, 2012. Assessment of yeast cell wall as replacements for antibiotic growth promoters in broiler diets: effects on performance, intestinal histo morphology and humoral immune responses. J Anim Physiol Anim Nutri, 96: 275-284

Goff DA, 2011. Antimicrobial stewardship: bridging the gap between quality care and cost. Curr Opin Infect Dis, 24: S11-S20

Grayzel SE, Bender JB, Glore RP, Gumley N, Sykes JE et al., 2015. Understanding companion animal practitioners' attitudes toward antimicrobial stewardship. J Am Vet Med Assoc, 247: 883-884

Hardefeldt LY, Gilkerson JR, Billman Jacobe H, Stevenson MA, Thursky K et al., 2018. Barriers to and enablers of implementing antimicrobial stewardship programs in veterinary practices. J Vet Int Med, 32: 1092-1099

Joseph J and Rodvold KA, 2008. The role of carbapenems in the treatment of severe nosocomial respiratory tract infections. Expert Opin Pharmacother, 9: 561-575

Kallen MC and Prins JM, 2017. A systematic review of quality indicators for appropriate antibiotic use in hospitalized adult patients. Infect Dis Rep, 9(1): 6821 
Murphy D, Ricci A, Auce Z, Beechinor JG, Bergendahl $\mathrm{H}$ et al., 2017. EMA and EFSA Joint Scientific Opinion on measures to reduce the need to use antimicrobial agents in animal husbandry in the European Union, and the resulting impacts on food safety (RONAFA). EFSA J, 15 (1): 1-245

Page S, Prescott J and Weese S, 2014. The 5Rs approach to antimicrobial stewardship. Vet Rec, 175: 207

Prescott JF and Boerlin P, 2016. Antimicrobial use in companion animals and good stewardship practice. Vet Rec, 179: 486-488

Samanta I, Joardar SN and Das PK, 2018. Biosecurity Strategies for Backyard Poultry: A Controlled Way for Safe Food Production. In Food Control and Biosecurity (pp481517), Academic Press
Samanta I, Joardar SN, Ganguli D, Das PK and Sarkar U, 2015. Evaluation of egg production after adoption of biosecurity strategies by backyard poultry farmers in West Bengal. Vet World, 8: 177

Swaggerty CL, Pevzner IY and Kogut MH, 2014. Selection for pro-inflammatory mediators yields chickens with increased resistance against Salmonella enterica serovar Enteritidis. Poult Sci, 93: 535-544

Torrallardona D, 2009. Spray dried animal plasma as an alternative to antibiotics in weanling pigs-a review. Asian-Australasian J Anim Sci, 23: 131-148

Toth NR, Chambers RM and Davis SL, 2010. Implementation of a care bundle for antimicrobial stewardship. Am J Hlth Sys Pharma, 67: 746-749

Article received on 20.09.2019 and accepted for publication on 14.11.2019 